\title{
La determinación de costos a través de la metodología abc/abm como opción estratégica en la industria de servicios portuarios
}

\section{The determination of costs through the abc / abm methodology as a strategic option in the port services industry}

\author{
Lucía Noemí Morocho Malla \\ Imorochom@psg.ucacue.edu.ec \\ Universidad Católica de Cuenca, Cuenca \\ Ecuador \\ https://orcid.org/0000-0002-2616-0657 \\ Cecilia Ivonne Narváez Zurita \\ inarvaez@ucacue.edu.ec \\ Universidad Católica de Cuenca, Cuenca \\ Ecuador: \\ https://orcid.org/0000-0002-7437-9880 \\ Juan Carlos Erazo Álvarez \\ jcerazo@ucacue.edu.ec \\ Universidad Católica de Cuenca, Cuenca \\ Ecuador \\ https://orcid.org/0000-0001-6480-2270
}

Recibido: 9 de septiembre de 2019

Aprobado: 24 de septiembre de 2019

\section{RESUMEN}

El mundo empresarial actual es cada día más competitivo y siempre procura estar a la vanguardia de mecanismos, que garanticen optimizar recursos y mejorar la rentabilidad, es por ello, que las empresas apuestan a tener una innovación sistemática de procesos y actividades que sean eficientes y eficaces en el manejo de costos; siendo el caso para la empresa Loadfrans, dedicada a la prestación de servicios portuarios, quien presenta dificultades en la gestión de costos y en la generación de un margen de rentabilidad apropiado. Por ello, para una mejor solución a la problemática existente, se plantea aplicar en Loadfrans un modelo de Gestión de Costeo ABC/ABM, debido a que es un sistema de costeo que permite obtener una información confiable y propicia para la toma de decisiones en este tipo de empresas. Cabe indicar, que esta investigación se 


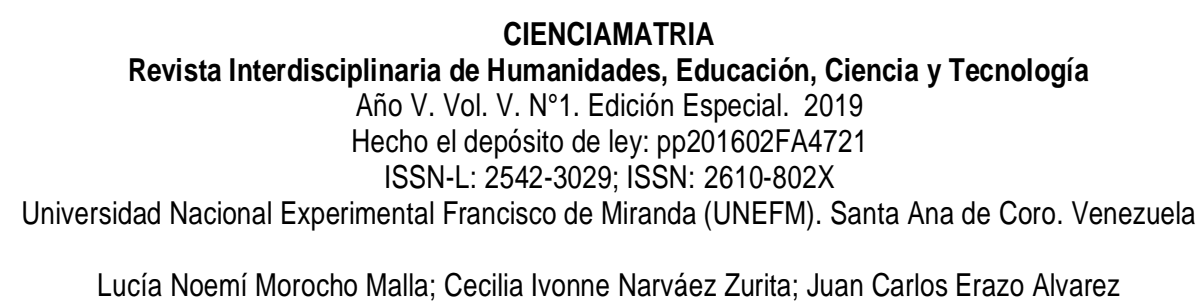

desarrolló bajo el estudio de un enfoque mixto, con un alcance descriptivo - explicativo; uno de los resultados más notables fue la obtención de los costos reales y margen de utilidad sugestiva de las operaciones portuarias de carga paletizada y suelta.

Descriptores: Costos; Herramientas; Gestión; Rentabilidad; Operaciones portuarias.

\begin{abstract}
The current business world is increasingly competitive and always seeks to be at the forefront of mechanisms that guarantee optimizing resources and improving profitability, which is why companies are committed to having a systematic innovation of processes and activities that are efficient and effective in cost management; being the case for the company Loadfrans, dedicated to the provision of port services, who presents difficulties in the management of costs and in the generation of an appropriate profit margin. Therefore, for a better solution to the existing problem, it is proposed to apply a $A B C$ / ABM Cost Management model in Loadfrans, because it is a costing system that allows obtaining reliable and favorable information for decision-making in this type of companies It should be noted that this research was developed under the study of a mixed approach, with a descriptive - explanatory scope; One of the most notable results was obtaining the real costs and profit margin suggestive of the palletized and loose port operations.
\end{abstract}

Descriptive: Cost; Tools; Management; Profitability; Port operations,

\title{
INTRODUCCIÓN
}

En la actualidad las empresas están sufriendo procesos de transformación a consecuencia de las altas exigencias de los clientes, menor duración del ciclo de vida de los productos, reducción de las inversiones en activos, disminución del peso de la mano de obra directa, incremento de los costos indirectos, precios bajos y mayor diferenciación de servicios y productos. Sin duda, estos factores surgen como consecuencia de la evolución técnico-científica que se gesta a la par de nuevos competidores a nivel mundial que obligan al sector empresarial a adoptar estrategias de excelencia encaminadas a producir nuevos e innovadores bienes y servicios, mejorar la funcionalidad de los productos y reducir los costos de producción.

Esto en definitiva se traduce según Lizana (2010), en "altos niveles de complejidad de las operaciones comerciales" (p.1), donde los sistemas de costeo tradicional que utilizan 


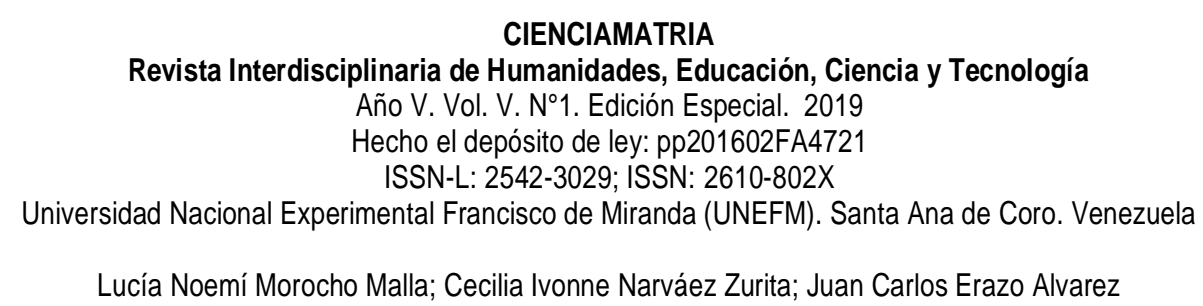

métricas enfocadas en la capacidad instalada no son idóneos para generar valor agregado e información útil para la gestión de la empresa con fines de control de las operaciones.

Es así que, a finales del siglo $\mathrm{XX}$ adquiere relevancia el costeo basado en actividades $(\mathrm{ABC})$, bajo un entorno dinámico en donde los directivos de las empresas manufactureras demandan información que aporte a la toma de decisiones relacionada con los procesos tecnológicos, con la combinación y diseño de productos y con la rentabilidad de la empresa. Sin embargo, es importante resaltar que los costos $A B C$ han tomado preeminencia en las empresas de servicios (Flores, Narváez, y Erazo, 2109) debido a la gran cantidad de costos indirectos en los que incurren y a los recursos que usan en cada uno de sus departamentos.

De acuerdo con lo expuesto, la operadora de servicios portuarios Loadfrans, localizada en el Puerto Bolívar, provincia de El Oro, Ecuador, requiere de un sistema de costeo dinámico que le permita determinar con exactitud el costo de los servicios que ofrece, así como la rentabilidad de los mismos, para ello resulta imprescindible conocer no únicamente los insumos y recursos que requiere sino también los departamentos relacionados, los procesos y las actividades productivas que los involucran. Por consiguiente, la presente investigación tiene por objetivo diseñar una metodología de costeo ABC y aplicarla a unos de los servicios portuarios que ofrece la empresa. Cabe puntualizar que la implementación de este sistema de costeo debe ir a la par del proceso de planeación estratégica.

\section{DESARROLLO}

El sistema de costeo tradicional caracterizado por la asignación de costos sobre la base de volúmenes elevados ha perdido vigencia ante el incremento de los gastos generales y disminución de la mano de obra directa en las empresas manufactureras, en definitiva, esto ha conllevado a que los administradores se interesen en conocer a profundidad los costos reales de su producción (Chacón, Bustos y Rojas, 2006). En tal sentido, la escasa 
información relacionada con las técnicas de innovación de control del costo de un producto o servicio ha generado la demanda de lineamientos contables, que aporten al conocimiento del costo de las transacciones desde un enfoque interno y externo; danto paso a técnicas dinámicas que permiten asignar costos de manufactura y costos ajenos a la manufactura a los productos (Brewer, Garrison, y Noreen, 2007).

De esta forma, la contabilidad de costos ha evolucionado considerablemente en las últimas décadas, dejando de lado el establecimiento de costos, el control financiero y la obtención de información financiera, para permitir el surgimiento de técnicas que persiguen la correcta asignación de los costos indirectos, la optimización de procesos, la generación de valor y las decisiones responsables (García, 2014; Marín y Martínez, 2006). Aunando a esta situación, contablemente se asume al costo como la suma total de las distribuciones en que incide un individuo para la adquisición de servicios o bienes, con el objetivo de que se genere un ingreso en el futuro, es aquí, donde la contabilidad de costos actúa como un proceso contable y estadístico destinado al conocimiento del costo del producto del bien o servicio que oferta la empresa.

Bajo este mismo contexto, García, Marín y Martínez (2006) consideran que la contabilidad de costos es la rama de la contabilidad dirigida a la medición de los recursos económicos consumidos; además, los autores asumen que esta rama de la contabilidad comprende un conjunto de procedimientos usados para medir, estudiar o estimar los costos, la rentabilidad y los rendimientos financieros de una empresa, con el propósito de elaborar y entregar información confiable, eficiente y eficaz a sus directivos, Rodríguez \& Puertas (2017). De modo que, la contabilidad de costos es considerada como una técnica empleada para llevar un debido manejo y control de recursos utilizados en la elaboración de un producto o prestación de servicios que genera un costo (Zapata, 2015), el mismo que se compone de varios elementos que deben ser considerados para determinar con precisión su valor. 


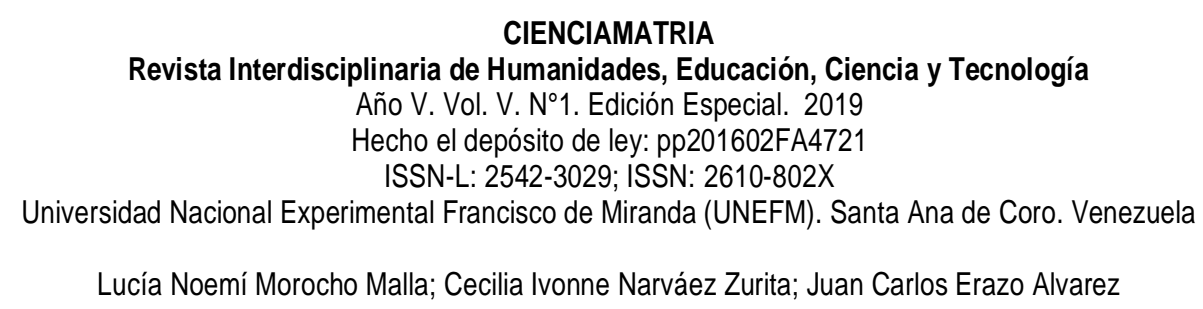

Dentro del costo de un producto o servicio se pueden divisar tres elementos:

1) Materia prima directa: se compone de aquellos recursos o materiales necesarios para la producción, sus características principales es que son identificables y relevantes.

2) Mano de obra: se considera así a todo esfuerzo físico o intelectual desarrollado por personas dentro del proceso de producción. El costo de este elemento es la remuneración que se reconoce al trabajador por el esfuerzo realizado.

3) Costos indirectos de fabricación (CIF): se caracterizan por ser fácilmente sustituidos, también conocidos como gastos generales de fabricación, por su naturaleza no forman parte del producto final, estos se dividen en materiales indirectos, mano de obra indirecta y otros costos indirectos de fabricación.

Estos elemento desempeñan un rol fundamental en el proceso productivo ya que para obtener un producto final atraviesan un proceso de transformación en donde se constituyen en productos en proceso, que para el efecto no se pueden dividir ni discriminar ya que se encuentran inmersos en la fabricación, producción o prestación del servicio, Ortega Polanco (2017).

Por otro lado, entre los distintos sistemas de costeo que considera la contabilidad (Morillo, 2002; Cuevas, Chávez, Castillo, Marino y Solarte, 2004; y, Cuervo, Duque y Osorio ,2013) se encuentran los siguientes:

- Costeo por órdenes de producción: el mismo consiste en calcular los costos por lotes u órdenes, este sistema se realiza mediante la elaboración de una orden de producción, donde se detalla la materia prima, la mano de obra y los costos indirectos de fabricación,

- Costeo por procesos: radica en la producción masiva, continúa y homogénea del mismo producto, los costos se calculan por período, al dividir la producción entre el periodo se obtienen los costos unitarios.

- Costeo directo según este método los costos variables de producción directos o indirectos se cargan a inventarios y forman parte del valor del producto final; los 


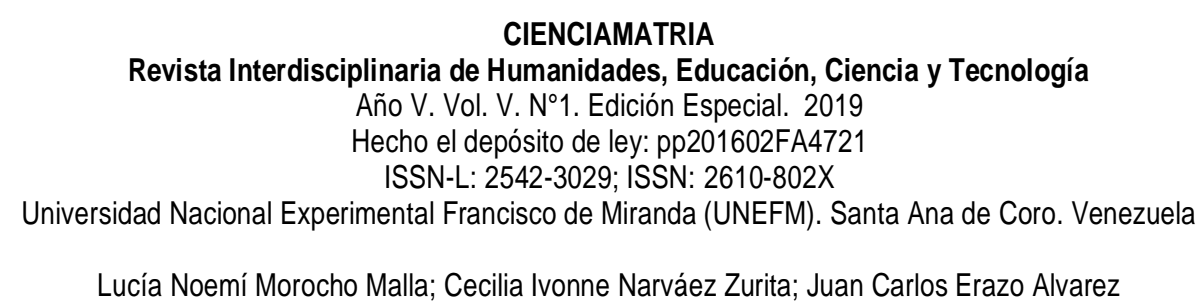

costos fijos de producción no son capitalizados, sólo se consideran gastos del período y se reconocen en el estado de resultados.

- Costeo total o de absorción, en este sistema todos los costos ya sean fijos o variables, directos o indirectos son cargados a la producción. Mientras se elabora la producción, los costos se capitalizan en los productos finalizados y forman parte del costo de dichos productos.

- Costeo por actividades ( $A B C)$, el sistema primero costean las actividades que demandan los productos o servicios y luego el costo de estas actividades se asignan usando tasas en forma escalonada, su relevancia radica el análisis de los costos por objeto de costo y en análisis del valor de cada actividad.

De los sistemas de costos revisados, el costeo basado en actividades es aplicable a cualquier tipo de organizaciones, dentro de las cuales se encuentran las empresas de servicios portuarios. Al respecto, Rodriguez (2008) considera que la gestión de costos ABC (Activity Based Costing) es un modelo basado en la agrupación de centros de costos que forman parte de una secuencia de valor de los servicios que oferta una empresa. Este sistema se basa en tres conceptos básicos que son: procesos, actividades y recursos.

Resulta así mismo interesante, analizar la perspectiva del ABM (Activity Based Management) ya que este sistema es importante para la gestión de costos de una empresa, debido a que consiste en agrupar y centrar la gestión de las actividades indirectas en múltiples niveles, más allá de la productividad o actividad directa, para alcanzar mayor optimización en el costo del bien recibido por el cliente y en el beneficio que trae consigo, así las empresas pueden encontrar valor en la información generada por el sistema de costeo y considerarla como herramienta útil en el proceso de toma de decisiones (Caldera y Ripoll, 2003).

A pesar de estas aportaciones, Sánchez (2013) infiere que es necesario diferenciar el $A B M$ y el $A B C$, ya que el $A B M$ está orientado a lograr una excelencia empresarial en el ámbito de la administración, mientras que, el $A B C$ posee una extensión contable y 


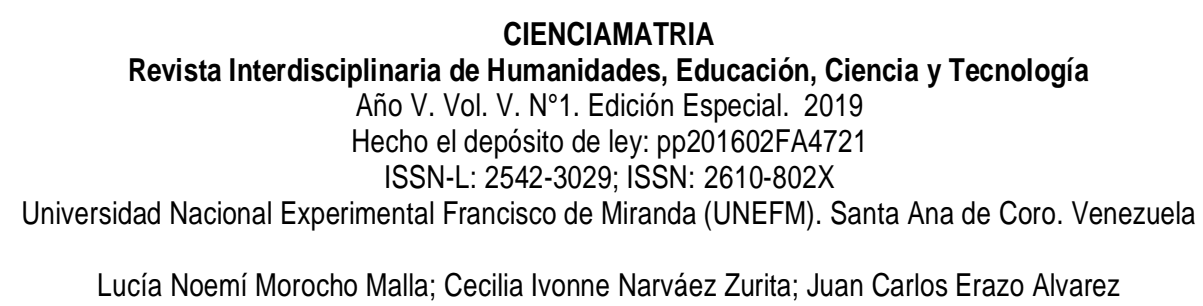

numérica, que apoya a los objetivos generales que tiene el ABM. De manera que, los dos sistemas buscan obtener información sobre las causas que ocasionan la realización de una actividad o trabajo y pretenden analizar la forma en la que se realizan ciertas medidas de costeo. Por lo tanto, se hace alusión al término $A B C / A B M$ para referirse a la metodología de cálculo y gestión de costos de las actividades, asumiendo que es un proceso integral y no aislado (Caldera y Ripoll, 2003), el mismo está construido sobre el eje vertical $(A B C)$ que proporciona información en un momento determinado, y el eje horizontal (ABM) que revela las causas y resultados de los costos. En tal sentido, los administradores deben manejar la información de ABM para instaurar prioridades, con enfoque en la reducción de costos y una apropiada toma de decisiones estratégicas (Cuevas, et al., 2004).

Por su parte, Ripoll, Caldera, y Vega (2006) indican que el sistema ABC/ABM es una herramienta enfocada en el cálculo, cuantificación y manejo de las actividades o tareas que lleva a cabo una empresa para desarrollar su actividad usual, aplicando al departamento correspondiente el costo afectado. En lo organizativo y jerárquico, sigue un orden en el cumplimiento de los canales regulares para la realización y desempeño de los costos que conforman las unidades organizativas. De acuerdo con esta gestión, los costos solos no son una fuente de valor competitivo, puesto que, únicamente las actividades son capaces de agregar valor, por ello, la gerencia debe controlar y eliminar la pérdida de esfuerzos inválidos con aquellas actividades que no agregan valor. La identificación de las causas de este desgaste en la gestión de actividades operacionales refleja el éxito de una empresa y constituye un arduo trabajo que lleva a la mejora continua.

Por otro lado, el ABC/ABM en la actualidad figura como uno de los sistemas más utilizados por las organizaciones debido a su moderna aplicación. De manera general, el modelo $\mathrm{ABC} / \mathrm{ABM}$ se lo debe implementar cuando el porcentaje de costos indirectos sobre el total de costos de la producción represente un valor significativo. Otro caso de aplicación se da cuando las empresas bajo intensas presiones de precios en el mercado, 


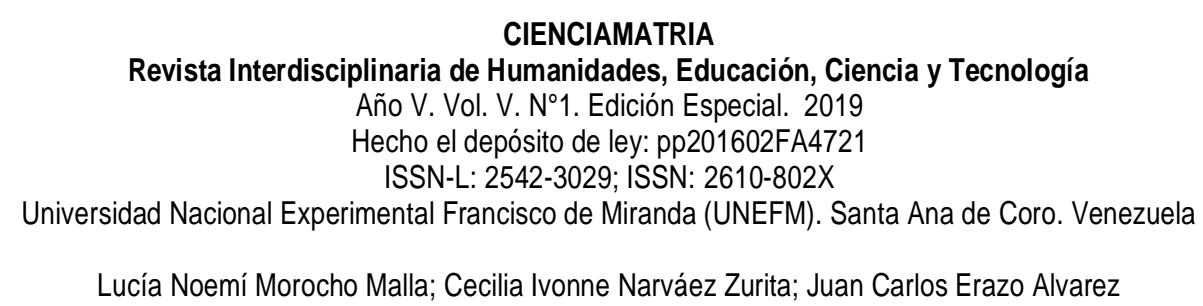

requieran conocer con exactitud la estructura del costo de los productos. Adicionalmente, un tercer caso es en organizaciones con una alta escala de productos con diferentes procedimientos en la fabricación, y en los que es difícil conocer la proporción de gastos indirectos de cada producto (Pérez y Tapanes, 2009). El proceso de implementación del sistema $A B C / A B M$ varía entre empresas de acuerdo a su capacidad y dimensión, ya que los recursos sobre todo en las pequeñas y medianas empresas son más limitados, y por ende su implementación debe ser eficiente y eficaz; para tal efecto, López, Gómez y Marín (2011) exponen la importancia de que las pequeñas empresas utilicen modelos sencillos de hojas de cálculo para distinguir los beneficios del $A B C$ sin la necesidad de desarrollar softwares complejos y costosos.

Ante la aplicación del método $\mathrm{ABC} / \mathrm{ABM}$, el principal factor de éxito percibido es el apoyo de la alta administración, para ello se registran elementos facilitadores como la cultura sistémica de adopción de nuevas formas de gestión, jerarquía de puestos, comunicación corporativa organizada y participativa, y alto compromiso de profesionales dedicados al proyecto (Rodríguez, 2010). Contrario a esto, también hay problemas ante la aplicación del $A B C / A B M$; por ejemplo, los altos costos vinculados a la constante actualización de los cambios de los inductores del costo o cost drives, la subsistencia de objetivos que no se adecúan a una perspectiva directa con el $A B C$, la perseverancia en la utilización de normas financieras y contables tradicionales, así como la resistencia al cambio ocasionada por la adopción de avanzados métodos en un corto período de tiempo (Ibídem, 2010).

\section{METODOLOGÍA PARA LA IMPLEMENTACIÓN DEL SISTEMA ABC/ABM}

Ante una adecuada aplicación de costos, se establece formular las fases del modelo de gestión $\mathrm{ABC} / \mathrm{ABM}$, a través de una correcta metodología de implementación; sobre este asunto, Cuervo, Duque, y Osorio (2013) exponen que las fases de implementación implican desde el conocimiento de la estructura de los costos hasta el seguimiento y evaluación final del modelo a realizar; ante ello cada etapa conlleva una cadena de 


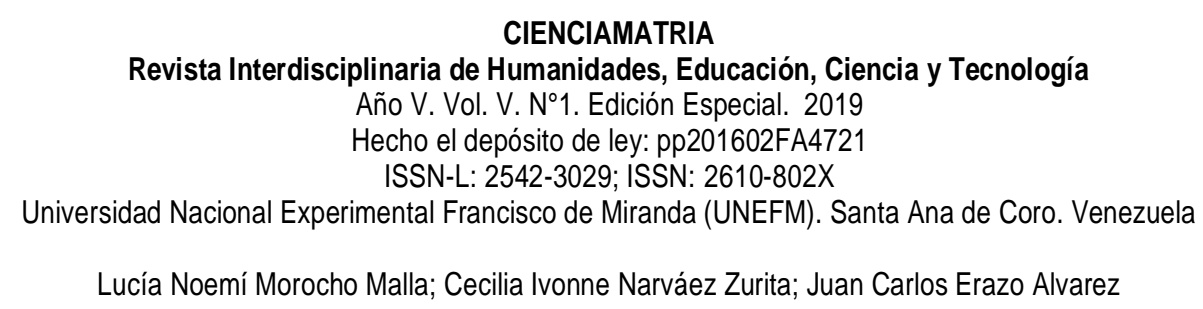

procesos que se deben tomar en consideración de manera concisa y concreta, en relación a qué se va ejecutar y el cómo se va aplicar dentro de una empresa. Para este fin se exponen a continuación siete fases:

- Fase uno: es importante tener conocimiento preliminar de la estructura de costos tomando en cuenta el esfuerzo, tiempo y dinero que se invierten para determinar, medir y controlar.

- Fase dos: radica en coordinar, orientar e identificar los productos principales y secundarios que se producen en el proceso. Para obtener información de los procesos se hace uso de técnicas de investigación como la entrevista y las encuestas.

- Fase tres: se fundamenta en que la metodología $A B C$ tiene como fin establecer las actividades que se ejecutan en cada uno de los procesos de los costos. Principalmente se elabora con un listado de todas las actividades y la manera en que se llevaran a cabo. Por lo general, se encargan de formular preguntas que servirán para una mejor aplicación de identificación de actividades, entre las interrogantes que se formulan están las siguientes: ¿Para qué?, la actividad busca respuesta sobre la importancia o para que esta direccionada; ¿En qué momento y dónde?, cada actividad pueden incurrir en otro lugar y los resultados pueden ser mejores en calidad, tiempo y costo; ¿Quién y cómo?: quien será la persona responsable, con que competencias y conocimientos cuenta, y cuál es el método a ser empleado.

- Fase cuatro: la etapa cuatro requiere ser primordial por la razón que agrupa las actividades que permiten simplificar la cantidad o nivel de detalle.

- Fase cinco: es aquí donde se establece los costos e inductores tanto para los recursos como para las actividades, por lo general, se pueden determinar por categoría y también se especifican por cada inductor referente a su estabilidad 


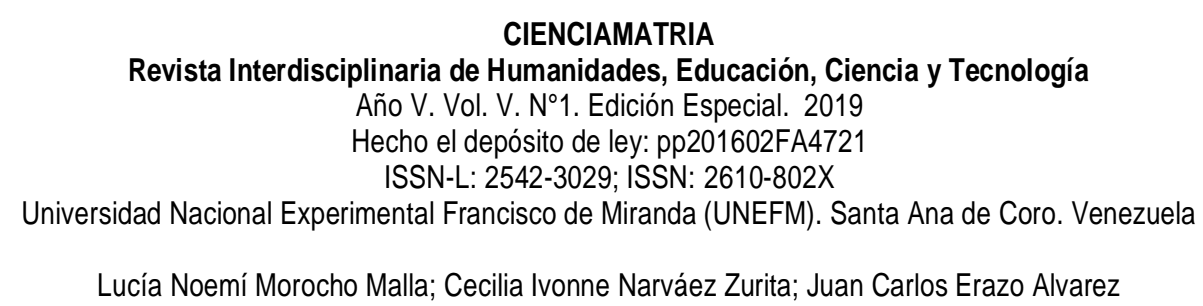

interna en la empresa, haciendo uso de cualquier método para la distribución de las actividades entre los productos.

- Fase seis: se determinan los objetos de costos, donde se conoce los productos que se elaboran y comercializan para identificar sus costos.

- Fase siete: después de haber establecido cada estándar, se implementa el modelo de costos; $y$, además, se desarrolla el seguimiento que engloba la evaluación del desempeño del modelo en donde se designan los responsables para cada actividad, al final de cada reporte se podrá mejorar el modelo identificando en donde se pueden incorporar nuevos procedimientos.

\section{RELACIÓN ENTRE LA GESTIÓN DE COSTOS ABC/ABM Y LA RENTABILIDAD}

La aplicación del modelo de gestión de costos $A B C / A B M$ mejora la calidad de los productos o servicios de las empresas, en costos y en tiempo de ciclo, incluyendo además la rentabilidad, debido a su influencia directa sobre las variables de ejecución. De esta manera, entendiendo el marco de la correlación entre $A B C / A B M$ y la rentabilidad, los encargados de las empresas podrán decidir en que basan la mejora de sus procesos operativos y consecuentemente en su rentabilidad (Rodríguez, 2010).

Sobre la base de lo expuesto, Morillo (2002) afirma que la rentabilidad es una medida concerniente a las utilidades, que resulta de la comparación de las utilidades netas obtenidas en la empresa con las ventas (rentabilidad o margen de utilidad neta sobre ventas), la inversión realizada (rentabilidad económica o del negocio), y los fondos aportados por sus propietarios (rentabilidad financiera o del propietario). Mientras que, Sánchez (2002) señala que la rentabilidad es un principio aplicable a toda operación económica en la que intervienen elementos materiales, humanos y financieros con el objetivo de obtener ciertos efectos. También se puede definir como el resultado total de las decisiones tomadas por la administración de una empresa (Hoz, Ferrer y Hoz, 2008). Según Coronado (2017), y que por lo general se expresan en porcentajes. 


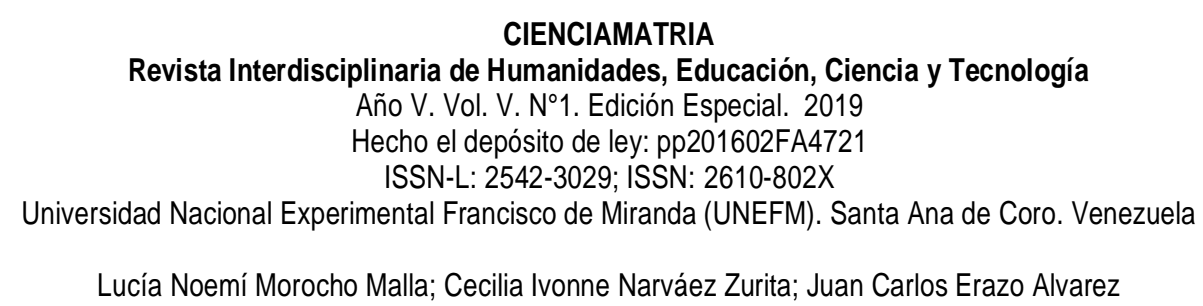

En la literatura especializada se encontró dos tipos de rentabilidad:

La rentabilidad económica que se constituye como un indicador primordial para calificar la eficiencia en la administración empresarial, ya que es el comportamiento de los activos que determina que una empresa sea o no rentable económicamente, en este contexto, el excluir la forma en que se han financiado los activos admitirá que se determine si una empresa no rentable es causada por dificultades en el progreso de su actividad o por una defectuosa política económica, siendo este tipo de rentabilidad, el cociente entre el beneficio antes de gastos financieros e impuestos y la inversión neta.

La rentabilidad financiera es un indicador que demuestra los beneficios obtenidos a consecuencia de invertir dinero, dicho en otras palabras, comprende el rendimiento alcanzado por las inversiones realizadas. Según los autores Hoz, Ferrer y Hoz (2008), la rentabilidad financiera es el cociente entre el beneficio neto y los recursos propios que emplea la empresa para la producción de un bien o servicio.

Dentro de los indicadores de rentabilidad, se demuestra que las inversiones rentables forman parte de la razón de ser de las empresas; y ante la necesidad de medir esto, es necesario su implementación porque permiten conocer la efectividad de sus operaciones y conversión de ventas en ganancias (Hoz, Fontalvo y Morelos, 2014; Pacheco, 2012). En este caso, es necesario considerar las siguientes características para medir la rentabilidad y así elaborar una ratio o indicador significativo:

- Debe existir un nexo causal entre los recursos o inversiones consideradas como denominador y el resultado o excedente al que se enfrentarán.

- En el establecimiento de la suma de recursos invertidos se considerará el promedio del periodo, debido a que es necesaria para ampliar la representación de los mismos.

- Es necesario también definir el periodo de tiempo de la medición de la rentabilidad (normalmente en el ejercicio contable), ya que en ocasiones de breves espacios, de tiempo o incorrectas fijaciones de períodos se suele incidir en errores. 
Finalmente, el estado de resultados es la primordial herramienta para evaluar la rentabilidad de una empresa en un determinado periodo, ya sea en semanas, meses o años. En este caso, el estado de resultados se debe mostrar por etapas, ya que, si se restan los ingresos, los gastos y costos del periodo, se logra distinguir la utilidad neta del bien o servicio. El estado de resultados es tan necesario como el balance general para la construcción de indicadores de rentabilidad, debido a que no sólo se precisa de información concerniente a los resultados, ingresos o ventas, sino también se debe disponer de información relacionada con la organización financiera, fundamentalmente con los activos y el capital invertido (Hoz, Ferrer y Hoz, 2008).

\section{METODOLOGÍA DE LA INVESTIGACIÓN}

Este estudio fue realizado bajo un enfoque mixto, por cuanto se aplicaron instrumentos que permitieron la recolección de información cuantitativa (encuestas) y cualitativa (entrevista y observación) en una única línea del tiempo (finalidad transversal) con un alcance descriptivo - explicativo, en donde se estableció un marco teórico sobre la gestión de costos ABC/ABM y se expuso la problemática de la empresa "LOADFRANS" S.A. a raíz de datos proporcionados por los integrantes de la población de estudio.

Se utilizó el método histórico - lógico mediante el cual se seleccionó la literatura respecto de las variables de investigación de acuerdo a la trascendencia, relevancia de la información y su pertinencia, también se empleó el método inductivo - deductivo, a través de la inducción se derivaron principios generales de la teoría de costos, a partir de observaciones especificas a investigaciones realizadas para determinar un patrón y establecer generalizaciones, mientras que la deducción permitió aplicar la teoría de costos $\mathrm{ABC} / \mathrm{ABM}$ a una situación específica de la empresa en estudio. Por último se usó el método analítico - sintético en la descomposición de las variables, para su estudio integral. El universo de estudio, estuvo integrado por 75 personas entre directivos, administrativos y operativos que se desempeñan en las distintas áreas de la empresa. 


\section{RESULTADOS}

Mediante la aplicación de los instrumentos de investigación se obtuvieron los siguientes resultados:

Políticas de financiamiento: la cartera crediticia de los clientes supera los treinta días de mora, lo que es contrario al tiempo determinado en las políticas internas de la empresa. Como consecuencia, existe menor liquidez necesaria para la ejecución factible de las operaciones.

Sistema de costeo: la empresa "LOADFRANS" S.A., es evidente que el sistema de costeo responde a prácticas tradicionales e incipientes. El problema más significativo que tiene la empresa es la complejidad con que determinan, manejan y controlan los costos de los servicios portuarios, ya que carecen de herramientas tecnológicas que les faciliten dicho proceso.

Costos de servicios: los costos que intervienen en la dinámica portuaria son la materia prima, la mano de obra directa y los costos indirectos de fabricación, de ellos se idéntico aspectos relevantes que permiten su determinación, control y asignación a los diferentes servicios. A pesar de que en la empresa se evidencia el cálculo de costos de los servicios, la metodología empleada para el manejo y asignación de los mismos no es la más idónea, ya que mayoritariamente lo realizan de manera empírica llegando a sub-costear en unos casos y sobre- costear en otros.

Herramientas de control: se constató que la empresa no cuenta con herramientas sofisticadas que permitan mantener un control óptimo de los costos que se incurren para la prestación de servicios generales, de carga, a la nave, conexos, de vigilancia y otros servicios que ofrece, por consiguiente, se imposibilita evaluar los resultados en función de los recursos utilizados. Cabe resaltar que a partir de los controles sobre los servicios y los costos se pueden establecer acciones que incidan favorablemente sobre el proceso de costeo. 


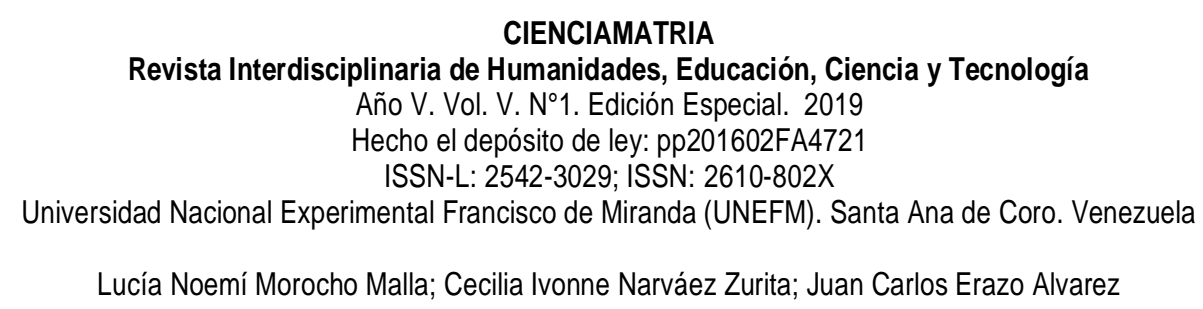

Planificación de operaciones: a empresa no maneja una adecuada planificación de las operaciones portuarias, lo que conlleva a generar costos y gastos altos sobre el personal, materiales y costos indirectos de fabricación.

Estrategias en base a costos y productividad: las estrategias se determinan en base a las metas a largo plazo, al direccionamiento estratégico y a los recursos necesarios, sin embargo, se observa en la empresa la ausencia de planificación presupuestaria que incida en la adquisición de insumos, ya que la compra de los mismos se realiza sin apoyo de controles e inventarios que determinen la cantidad de pedido, faltantes y sobrantes, en tal sentido, no existen estrategias en base al volumen de servicios ofertados.

En este orden de ideas se concluye que una parte importante de esta empresa presenta debilidades en cuando a la gestión de costos, no obstante, dadas las limitaciones que poseen aplican técnicas tradicionales para cuantificarlos y controlarlos. En base a estos resultados, se determina la necesidad de aplicar un modelo de gestión de costos ABC/ABM en la empresa Loadfrans S.A. , con la finalidad de costear cada uno de los servicios y operaciones portuarias, y determinar la rentabilidad de las mismas. El esquema del modelo contiene 7 componentes que se encuentran sistematicamente interrelacionados (ver figura 1).

\section{Figura 1.}

Esquema del modelo de gestión de costos $A B C / A B M$ para las operaciones portuarias de la empresa Loadfrans S.A. de la ciudad de Machala 


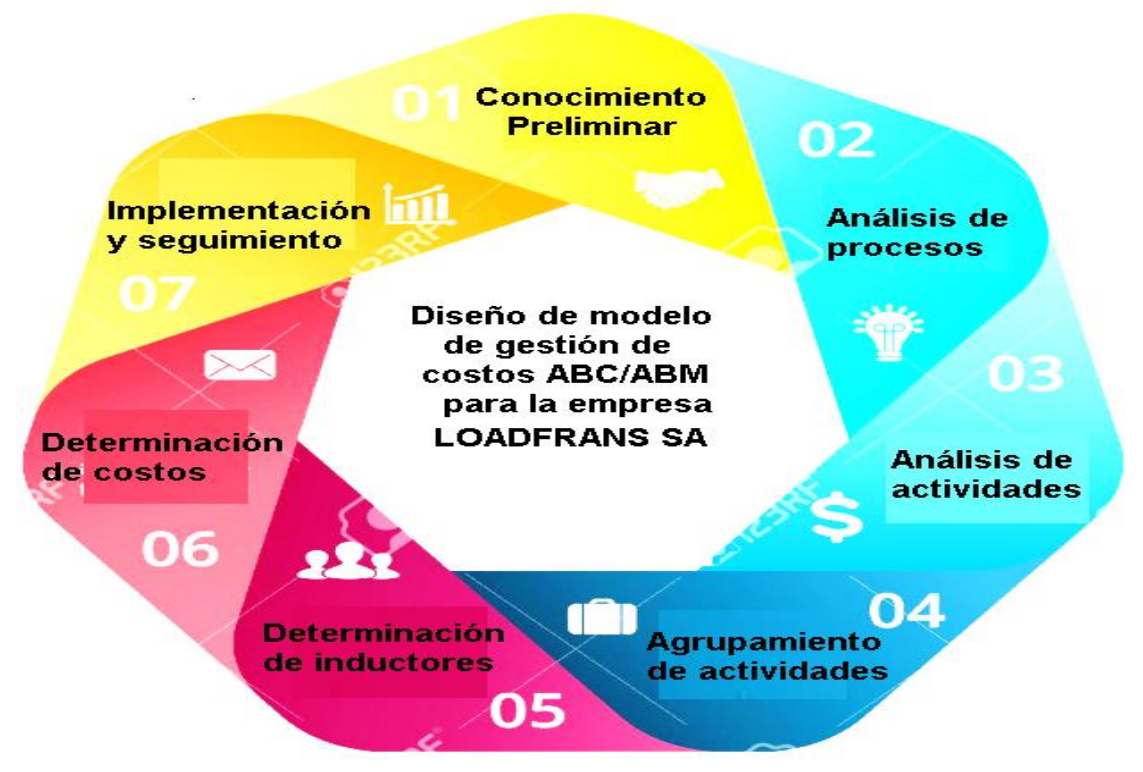

Conocimiento preliminar: para el desarrollo del modelo de gestión basado en actividades $A B C / A B M$ se identificó el proceso operativo que maneja la empresa conformado por la materia prima, mano de obra directa y los procesos que se han relacionado de forma directa con la actividad de la compañía. El servicio operativo de carga se encuentra definido desde el requerimiento de la materia prima a bodega, luego la utilización del recurso humano, y la aplicación de los costos indirectos.

El siguiente proceso a realizarse, concierne al diseño estratégico creando la razón de ser de la empresa a través de la misión y visión. Al hablar de misión se enfatiza en la parte activa del buen desarrollo de la prestación de servicios portuarios bajo las diferentes normas de calidad y de gestión ambiental, y como visión la empresa Loadfrans apuesta en llegar a ser reconocida a nivel nacional e internacional en los puertos marítimos multipropósitos de mayor afluencia del mundo.

Se plantea analizar dos tipos de carga, la carga suelta y carga paletizada para la empresa Loadfrans, donde se identifican los elementos de los costos directos e indirectos. 
CIENCIAMATRIA

Revista Interdisciplinaria de Humanidades, Educación, Ciencia y Tecnología

Año V. Vol. V. №1. Edición Especial. 2019

Hecho el depósito de ley: pp201602FA4721

ISSN-L: 2542-3029; ISSN: 2610-802X

Universidad Nacional Experimental Francisco de Miranda (UNEFM). Santa Ana de Coro. Venezuela

Lucía Noemí Morocho Malla; Cecilia Ivonne Narváez Zurita; Juan Carlos Erazo Alvarez

En la tabla 1 y 2, se describen los costos directos e indirectos para derivar la distribución de las actividades existentes por los CIF. En la tabla 1, el costo unitario obtenido a través del costo tradicional para las operaciones portuarias de carga suelta es de $\$ 0,111$.

Tabla 1

Costos directos e indirectos de la Empresa Loadfrans de carga suelta

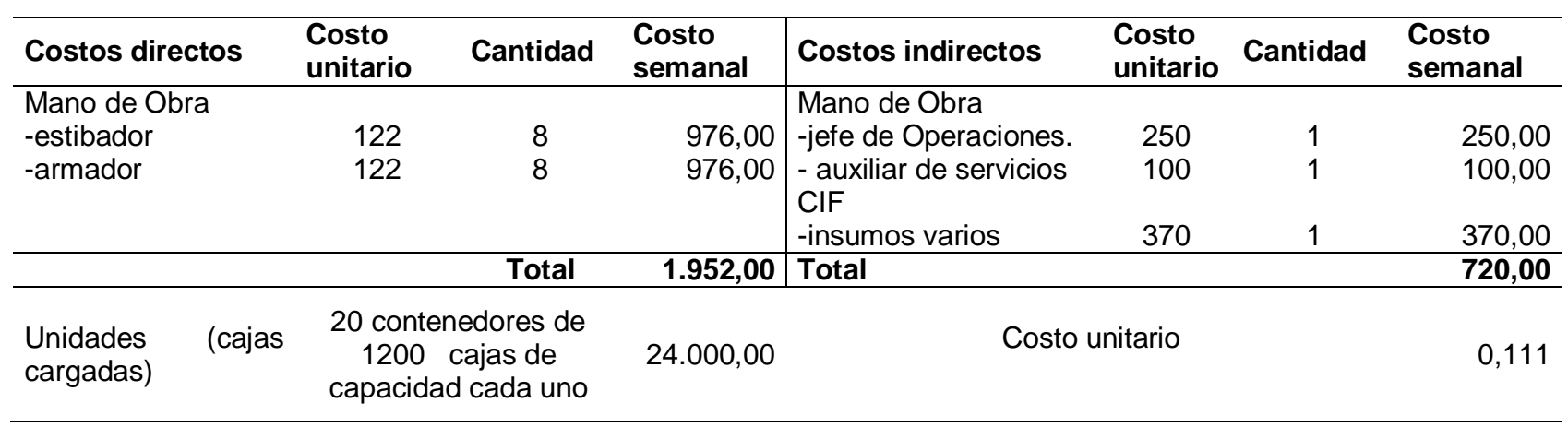

Fuente. Loadfrans

Una vez analizado los costos directos e indirectos de carga suelta, se procede a verificar los costos directos e indirectos de carga paletizada a través del mismo método tradicional. En la tabla 2, se determina el costo unitario de $\$ 0,403$ por las operaciones portuarias de carga paletizada, tanto en la tabla 1 y 2 se puede verificar que bajo el método tradicional aplicado, no es posible identificar el departamento donde se encuentran mayor incidencia en los costos.

\section{Tabla 2}

Costos directos e indirectos de la Empresa Loadfrans de carga paletizada

\begin{tabular}{|c|c|c|c|c|c|c|c|}
\hline Costos directos & $\begin{array}{l}\text { Costo } \\
\text { unitario }\end{array}$ & Cantidad & $\begin{array}{l}\text { Costo } \\
\text { semanal }\end{array}$ & \begin{tabular}{|l|} 
Costos \\
indirectos
\end{tabular} & $\begin{array}{l}\text { Costo } \\
\text { unitario }\end{array}$ & Cantidad & $\begin{array}{l}\text { Costo } \\
\text { semanal }\end{array}$ \\
\hline $\begin{array}{l}\text { Materia prima } \\
\text { - pallets de madera }\end{array}$ & & & & Mano de Obra & & & \\
\hline $\begin{array}{l}48 \text { cajas por pallets } \\
\text { equivalente a } 20 \text { pallets por } \\
\text { contenedor) } \\
\text {-Esquineros de plástico }\end{array}$ & 10,80 & 400 & $4.320,00$ & $\begin{array}{l}\text {-jefe de } \\
\text { Operaciones. }\end{array}$ & 230,00 & 1 & 230,00 \\
\hline $\begin{array}{l}(4 \text { esquineros por pallets } \\
\text { equivalente a } 20 \text { pallets por } \\
\text { contenedor) }\end{array}$ & 0,48 & 1600 & 768,00 & -jefe mecánico. & 170,00 & 1 & 170,00 \\
\hline -refuerzos de plásticos & 0,07 & 3200 & 224,00 & $\begin{array}{l}\text { - auxiliar de } \\
\text { servicios }\end{array}$ & 95,00 & 1 & 95,00 \\
\hline
\end{tabular}


CIENCIAMATRIA

Revista Interdisciplinaria de Humanidades, Educación, Ciencia y Tecnología

Año V. Vol. V. N¹. Edición Especial. 2019

Hecho el depósito de ley: pp201602FA4721

ISSN-L: 2542-3029; ISSN: 2610-802X

Universidad Nacional Experimental Francisco de Miranda (UNEFM). Santa Ana de Coro. Venezuela

Lucía Noemí Morocho Malla; Cecilia Ivonne Narváez Zurita; Juan Carlos Erazo Alvarez

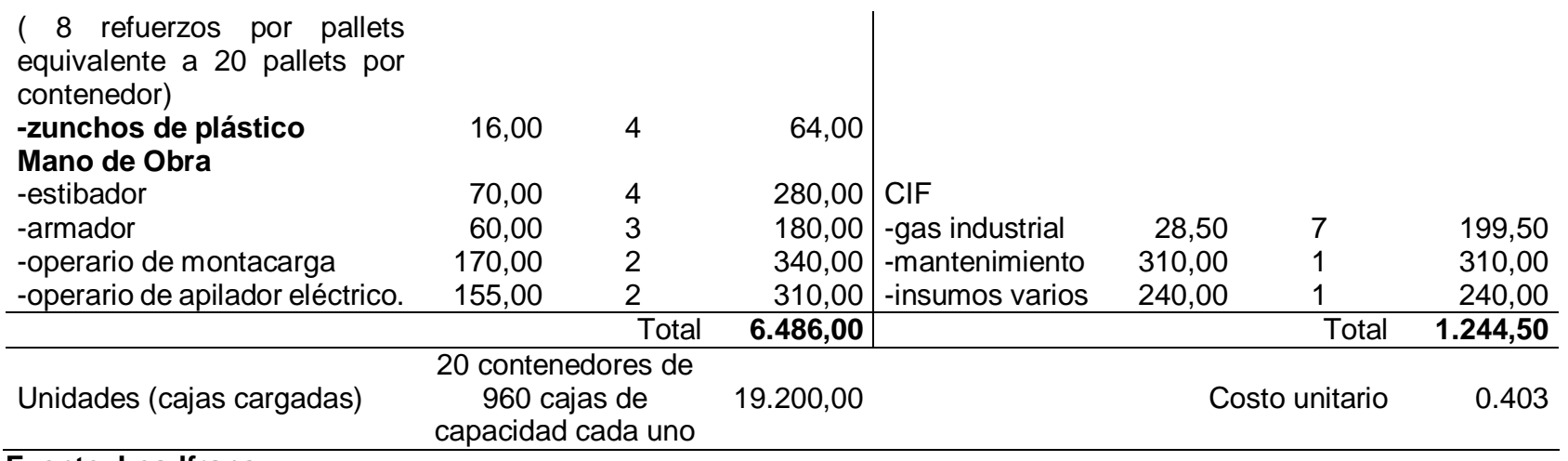

Fuente. Loadfrans

Análisis de procesos: en esta fase se identifica el nivel de jerarquía de cada actividad que realiza la empresa y su relación con los centros de costos (ver figura 2).

\section{Figura 2}

Procesos y actividades de la empresa

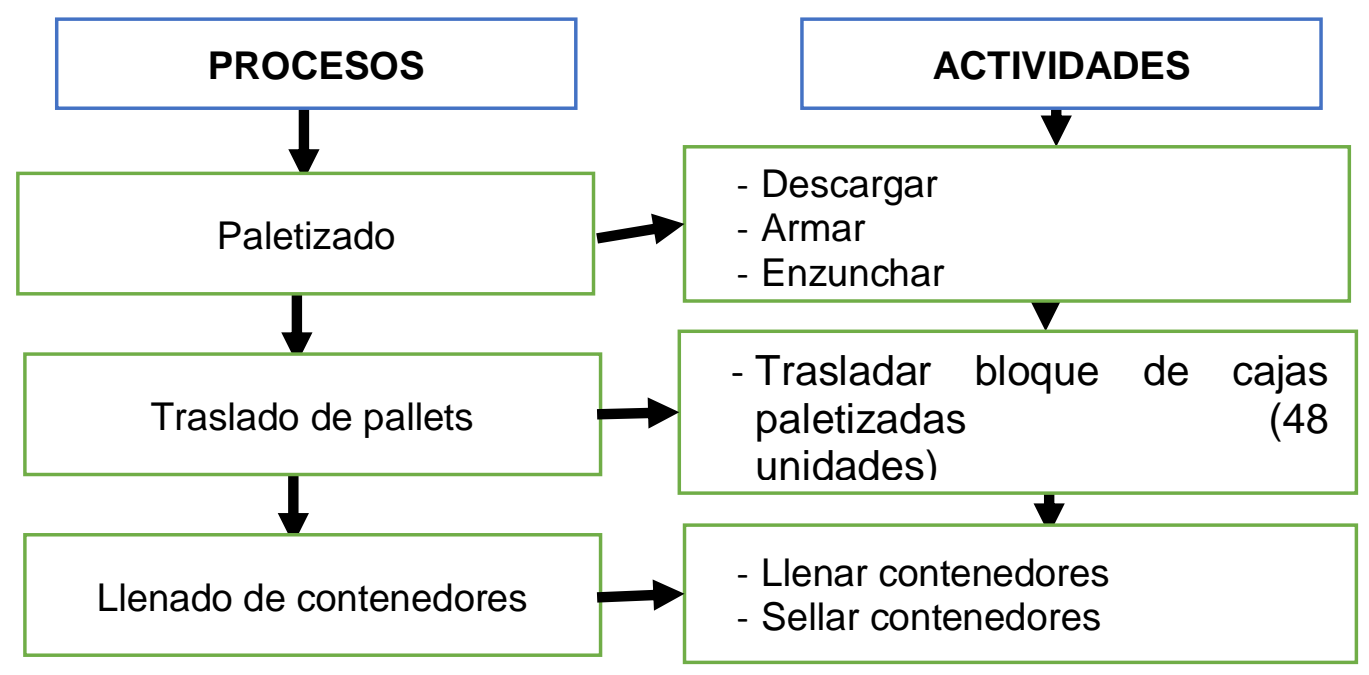

Análisis de actividades: Una vez realizada la jerarquización, se continua con el siguiente proceso y actividades de la empresa, en cuanto a la elección y determinación de los inductores de recursos, y por ende a establecer los costos indirectos de cada actividad, tal como se expresa en la tabla 3 y 4. 
Tabla 3

Centro de costos y actividades de carga paletizada

\begin{tabular}{|c|c|c|c|}
\hline Centro de costos & Actividades & Inductores & Responsables \\
\hline \multirow{4}{*}{ Paletizado } & Descargar & horas hombre & Estibador \\
\hline & Armar & horas hombre & Armador \\
\hline & Enzunchar & horas hombre & Armador \\
\hline & Reforzar & horas hombre & Armador \\
\hline $\begin{array}{c}\text { Traslado de pallets } \\
\text { armado }\end{array}$ & Trasladar & horas hombre & $\begin{array}{l}\text { Operador de } \\
\text { montacaraas }\end{array}$ \\
\hline $\begin{array}{l}\text { Llenado de } \\
\text { contenedores }\end{array}$ & Llenar & horas hombre & $\begin{array}{c}\text { Operador de } \\
\text { apilador eléctrico }\end{array}$ \\
\hline $\begin{array}{l}\text { Cierre de contenedor y } \\
\text { sellado }\end{array}$ & $\begin{array}{l}\text { Cerrar } \\
\text { Sellar }\end{array}$ & $\begin{array}{l}\text { horas hombre } \\
\text { horas hombre }\end{array}$ & $\begin{array}{l}\text { Jefe de } \\
\text { operaciones }\end{array}$ \\
\hline
\end{tabular}

Fuente. Loadfrans

Tabla 4

Centro de costos y actividades de carga suelta

\begin{tabular}{cccc}
\hline Centro de costos & Actividades & Inductores & Responsables \\
\hline Traslado de cajas & Trasladar & horas hombre & Estibador \\
Llenado de & Llenar & horas hombre & Llenador \\
contenedores. & Cerrar & horas hombre & Jefe de \\
Cierre de contenedor y & Sellar & horas hombre & operaciones \\
sellado &
\end{tabular}

Fuente. Loadfrans

Determinación de inductores: El cálculo de unidades para la presente investigación, se determina por dos tipos de carga existentes en la empresa Loadfrans:

- Carga Paletizada, es sobre 19.200 cajas que equivale a 20 contenedores con una capacidad de 960 unidades de caja de banano tipo 22XU cada uno.

- Carga suelta, por 24.000 cajas que corresponde a 20 contenedores, que contienen una capacidad de 1.200 unidades de cajas de banano tipo 22XU por cada contenedor. 
CIENCIAMATRIA

Revista Interdisciplinaria de Humanidades, Educación, Ciencia y Tecnología

Año V. Vol. V. №1. Edición Especial. 2019

Hecho el depósito de ley: pp201602FA4721

ISSN-L: 2542-3029; ISSN: 2610-802X

Universidad Nacional Experimental Francisco de Miranda (UNEFM). Santa Ana de Coro. Venezuela

Lucía Noemí Morocho Malla; Cecilia Ivonne Narváez Zurita; Juan Carlos Erazo Alvarez

Tabla 5

Distribución del CIF a las actividades de carga suelta

\begin{tabular}{|c|c|c|c|c|c|c|}
\hline \multirow[t]{2}{*}{ Costos indirectos } & \multirow[t]{2}{*}{$\begin{array}{c}\text { Costo } \\
\text { semanal }\end{array}$} & \multirow[t]{2}{*}{ Inductores } & \multirow[t]{2}{*}{ Unidad } & \multirow{2}{*}{$\begin{array}{c}\begin{array}{c}\text { Llenado de } \\
\text { contenedores }\end{array} \\
\text { Llenar } \\
\end{array}$} & \multicolumn{2}{|c|}{$\begin{array}{c}\text { Cierre de } \\
\text { contenedor y } \\
\text { sellado }\end{array}$} \\
\hline & & & & & Cerrar & Sellar \\
\hline \multicolumn{7}{|l|}{ Mano de Obra Indirecta } \\
\hline jefe de operaciones & 220,00 & \begin{tabular}{|l|} 
horas \\
hombre
\end{tabular} & 6 & & 110,00 & 110,00 \\
\hline auxiliar de servicios & 90,00 & $\begin{array}{l}\text { horas } \\
\text { hombre }\end{array}$ & 6 & 90,00 & & \\
\hline \multicolumn{7}{|l|}{ Materiales Indirectos } \\
\hline insumos varios & 350,00 & Unidad & 1.25 & 200,00 & 100,00 & 50,00 \\
\hline Total & 660,00 & & & 290,00 & 210,00 & 160,00 \\
\hline Cajas & 24.000 & & & & & \\
\hline Costo Unitario & 0,028 & & & & & \\
\hline
\end{tabular}

Fuente. Loadfrans

En la tabla 5 y 6 , se encuentran determinados los CIF por medio de los inductores de costos a cada actividad.

Tabla 6

Distribución del CIF a las actividades de carga paletizada

\begin{tabular}{|c|c|c|c|c|c|c|c|c|}
\hline \multirow[t]{2}{*}{ Costos indirectos } & \multirow[t]{2}{*}{$\begin{array}{l}\text { Costo } \\
\text { semanal }\end{array}$} & \multirow[t]{2}{*}{ Inductores } & \multirow[t]{2}{*}{ Unid. } & \multirow{2}{*}{$\begin{array}{l}\text { Paletizado } \\
\text { Descargar }\end{array}$} & \multirow{2}{*}{$\begin{array}{c}\text { Traslado } \\
\text { de pallets } \\
\text { armado } \\
\text { Trasladar }\end{array}$} & \multirow{2}{*}{$\begin{array}{c}\text { Llenado } \\
\text { de } \\
\text { contene } \\
\text { dores } \\
\text { Llenar }\end{array}$} & \multicolumn{2}{|c|}{$\begin{array}{l}\text { Cierre de } \\
\text { contenedor y } \\
\text { sellado }\end{array}$} \\
\hline & & & & & & & Cerrar & Sellar \\
\hline \multicolumn{9}{|l|}{ Mano de Obra } \\
\hline \multicolumn{9}{|l|}{ Indirecta } \\
\hline $\begin{array}{l}\text { jefe de } \\
\text { operaciones }\end{array}$ & 220,00 & horas hombre & 5 & & & & \multirow{4}{*}{110,00} & \multirow{3}{*}{110,00} \\
\hline jefe mecánico & 160,00 & horas hombre & 3 & & 100,00 & 60,00 & & \\
\hline $\begin{array}{l}\text { auxiliar de } \\
\text { servicios }\end{array}$ & 90,00 & horas hombre & 2 & 90,00 & & & & \\
\hline \multicolumn{8}{|l|}{$\begin{array}{l}\text { Materiales } \\
\text { Indirectos }\end{array}$} & \\
\hline gas industrial & 199,50 & Unidad & 7 & & 199,50 & & & \\
\hline Mantenimiento & 300,00 & horas hombre & 3 & & 150,00 & 150,00 & & \\
\hline insumos varios & 230,00 & horas hombre & 2 & & 100,00 & 80,00 & 20,00 & 30,00 \\
\hline Total & $1.199,50$ & & & 90,00 & 549,50 & 290,00 & 130,00 & 140,00 \\
\hline Cajas paletizadas & 19.200 & & & & & & & \\
\hline Costo Unitario & 0,062 & & & & & & & \\
\hline
\end{tabular}


Determinación de costos: en el último proceso, se establece el cálculo del costo de las actividades primarias y secundarias, luego de haber determinado cada actividad, se calcula el costo por el servicio ofertado de carga de cajas paletizadas y carga suelta en la tabla 7 y 8.

Tabla 7.

Determinación del costo por el servicio ofertado de paletizado de cajas

\begin{tabular}{|c|c|c|c|c|}
\hline Centro de costos & Actividades & Costos directos & $\begin{array}{c}\text { Costos } \\
\text { indirectos }\end{array}$ & $\begin{array}{c}\text { costos } \\
\text { total }\end{array}$ \\
\hline \multirow{4}{*}{ Paletizado } & Descargar & 260,00 & 90,00 & 350,00 \\
\hline & Armar & $4.485,00$ & & $4.485,00$ \\
\hline & Enzunchar & 64,00 & & 64,00 \\
\hline & Reforzar & 992,00 & & 992,00 \\
\hline \multirow{2}{*}{$\begin{array}{l}\text { Traslado de pallets } \\
\text { armado } \\
\text { Llenado de } \\
\text { contenedores }\end{array}$} & Trasladar & 320,00 & 549,50 & 869,50 \\
\hline & Llenar & 290,00 & 290,00 & 580,00 \\
\hline \multirow{3}{*}{$\begin{array}{l}\text { Cierre contenedores y } \\
\text { sellado }\end{array}$} & Cerrar & & 130,00 & 130,00 \\
\hline & Sellar & & 140,00 & 140,00 \\
\hline & Total & $6.411,00$ & $1.199,50$ & $7.610,50$ \\
\hline Unidades & 19.200 & 0,33 & 0,062 & 0,396 \\
\hline
\end{tabular}

Fuente: Loadfrans

Tabla 8

Determinación del costo por el servicio ofertado de cajas de carga suelta

\begin{tabular}{|c|c|c|c|c|}
\hline Centro de costos & Actividades & $\begin{array}{c}\text { Costos } \\
\text { directos }\end{array}$ & $\begin{array}{c}\text { Costos } \\
\text { indirectos }\end{array}$ & $\begin{array}{c}\text { costos } \\
\text { total }\end{array}$ \\
\hline \multirow{2}{*}{$\begin{array}{l}\text { Traslado de cajas } \\
\text { Llenado de } \\
\text { contenedores }\end{array}$} & Trasladar & 960,00 & & 960,00 \\
\hline & Llenar & 960,00 & 290,00 & $1.250,00$ \\
\hline \multirow{3}{*}{$\begin{array}{l}\text { Cierre contenedores y } \\
\text { sellado }\end{array}$} & Cerrar & & 210,00 & 210,00 \\
\hline & Sellar & & 160,00 & 160,00 \\
\hline & Total & $1.920,00$ & 660,00 & $2.580,00$ \\
\hline Unidades & 24.000 & 0,08 & 0,028 & 0,108 \\
\hline
\end{tabular}

Fuente. Loadfrans 
CIENCIAMATRIA

Revista Interdisciplinaria de Humanidades, Educación, Ciencia y Tecnología

Año V. Vol. V. N¹. Edición Especial. 2019

Hecho el depósito de ley: pp201602FA4721

ISSN-L: 2542-3029; ISSN: 2610-802X

Universidad Nacional Experimental Francisco de Miranda (UNEFM). Santa Ana de Coro. Venezuela

Lucía Noemí Morocho Malla; Cecilia Ivonne Narváez Zurita; Juan Carlos Erazo Alvarez

En la tabla 7 y 8 el costo unitario y costo total es menor al costo obtenido por el método tradicional, para mayor apreciación se presenta a continuación una hoja consolidada del costo total y costo unitario, obtenido a través de Costeo Total ABC/ABM y los costos por orden de producción que son manejados de forma empírica por la empresa.

Tabla 9

Hoja consolidada de costos total y unitario de carga suelta.

\begin{tabular}{|c|c|c|c|c|c|c|c|}
\hline \multirow{2}{*}{$\begin{array}{l}\text { Centro de costos } \\
\text { Actividades }\end{array}$} & \multirow{2}{*}{ 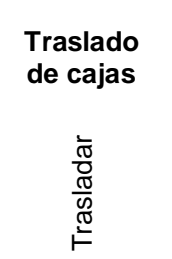 } & \multirow{2}{*}{ 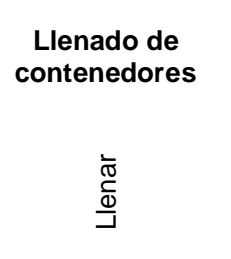 } & \multicolumn{2}{|c|}{$\begin{array}{l}\text { Cierre de } \\
\text { contenedor y } \\
\text { sellado }\end{array}$} & \multirow{2}{*}{ 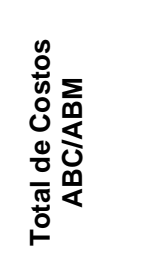 } & \multirow{2}{*}{ 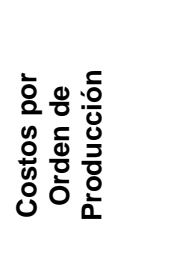 } & \multirow{2}{*}{ 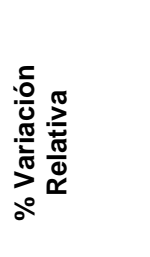 } \\
\hline & & & 㐫 & $\frac{\overline{\bar{\sigma}}}{\overline{\bar{D}}}$ & & & \\
\hline Costos Directos & 960,00 & 960,00 & 0,00 & 0,00 & $1.920,00$ & $1.952,00$ & $1.64 \%$ \\
\hline Materiales Directos & 0,00 & 0,00 & 0,00 & 0,00 & 0,00 & 0,00 & $0.00 \%$ \\
\hline Mano de Obra & 960,00 & 960,00 & 0,00 & 0,00 & $1.920,00$ & $1.952,00$ & $1.64 \%$ \\
\hline Estibador & 960,00 & & & & 960,00 & 976,00 & $1.64 \%$ \\
\hline Armador & & 960,00 & & & 960,00 & 976,00 & $1.64 \%$ \\
\hline Costos Indirectos & 0,00 & 290,00 & 210,00 & 160,00 & 660,00 & 720,00 & $8.33 \%$ \\
\hline $\begin{array}{l}\text { Mano de Obra } \\
\text { Indirecta }\end{array}$ & 0,00 & 90,00 & 110,00 & 110,00 & 310,00 & 350,00 & $11.43 \%$ \\
\hline Jefe de Operaciones & & & 110,00 & 110,00 & 220,00 & 250,00 & $12.00 \%$ \\
\hline Auxiliar de servicios & & 90,00 & & & 90,00 & 100,00 & $10.00 \%$ \\
\hline CIF & 0,00 & 200,00 & 100,00 & 50,00 & 350,00 & 370,00 & $5.41 \%$ \\
\hline insumos varios & & 200,00 & 100,00 & 50,00 & 350,00 & 370,00 & $5.41 \%$ \\
\hline Costo Total & $\begin{array}{r}960,00 \\
\text { Unidades }\end{array}$ & $\begin{array}{r}1.250,00 \\
24.000\end{array}$ & $\begin{array}{l}210,00 \\
\text { Costo L }\end{array}$ & $\begin{array}{l}160,00 \\
\text { Jnitario }\end{array}$ & $\begin{array}{r}2.580,00 \\
0,108\end{array}$ & $\begin{array}{r}2.672,00 \\
0,111\end{array}$ & $\begin{array}{r}3.44 \% \\
3 \%\end{array}$ \\
\hline
\end{tabular}

Tabla 10

Hoja consolidada de costos total y unitario de carga paletizada

\begin{tabular}{|c|c|c|c|c|c|c|c|c|c|c|c|}
\hline \multirow{2}{*}{$\begin{array}{l}\text { Centro de costos } \\
\text { Actividades }\end{array}$} & \multirow[b]{2}{*}{ 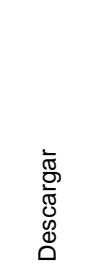 } & \multicolumn{3}{|c|}{ Paletizado } & \multirow{2}{*}{$\begin{array}{c}\text { Traslado } \\
\text { de pallets } \\
\text { armado } \\
\\
\frac{\bar{\pi}}{\frac{\pi}{\pi}} \\
\frac{\pi}{\mathbb{N}} \\
\stackrel{\widetilde{\pi}}{\models}\end{array}$} & \multirow{2}{*}{$\begin{array}{c}\begin{array}{c}\text { Llenado } \\
\text { de } \\
\text { contene } \\
\text { dores }\end{array} \\
\frac{\bar{\sigma}}{\bar{\varpi}}\end{array}$} & \multicolumn{2}{|c|}{$\begin{array}{c}\text { Cierre de contenedor } \\
\text { y sellado }\end{array}$} & \multirow{2}{*}{$\begin{array}{l}\text { Total de } \\
\text { Costos } \\
\text { ABC/ABM }\end{array}$} & \multirow{2}{*}{ 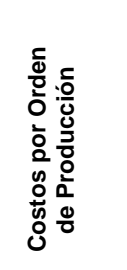 } & \multirow{2}{*}{ 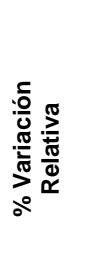 } \\
\hline & & $\stackrel{\bar{\varpi}}{\sum^{\frac{\pi}{\alpha}}}$ & 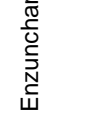 & 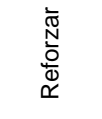 & & & 㐫 & $\frac{\frac{1}{0}}{\bar{N}}$ & & & \\
\hline Costos Directos & 260,00 & $4.485,00$ & 64,00 & 992,00 & 320,00 & 290,00 & 0,00 & 0,00 & $6.411,00$ & $6.486,00$ & $1.16 \%$ \\
\hline Materiales Directos & 0,00 & $4.320,00$ & 64,00 & 992,00 & 0,00 & 0,00 & 0,00 & 0,00 & $5.376,00$ & $5.376,00$ & $0.00 \%$ \\
\hline Materia prima & 0,00 & $4.320,00$ & 64,00 & 992,00 & 0,00 & 0,00 & 0,00 & 0,00 & $5.376,00$ & $5.376,00$ & $0.00 \%$ \\
\hline pallets de madera & & $4.320,00$ & & & & & & & $4.320,00$ & $4.320,00$ & $0.00 \%$ \\
\hline zunchos de plástico & & & 64,00 & & & & & & 64,00 & 64,00 & $0.00 \%$ \\
\hline esquinero de plástico & & & & 768,00 & & & & & 768,00 & 768,00 & $0.00 \%$ \\
\hline refuerzos de plástico & & & & 224,00 & & & & & 224,00 & 224,00 & $0.00 \%$ \\
\hline Mano de Obra & 260,00 & 165,00 & 0,00 & 0,00 & 320,00 & 290,00 & 0,00 & 0,00 & $1.035,00$ & $1.110,00$ & $6.76 \%$ \\
\hline Estibador & 260,00 & & & & & & & & 260,00 & 280,00 & $7.14 \%$ \\
\hline Armador & & 165,00 & & & & & & & 165,00 & 180,00 & $8.33 \%$ \\
\hline operario de montacarga & & & & & 320,00 & & & & 320,00 & 340,00 & $5.88 \%$ \\
\hline
\end{tabular}


CIENCIAMATRIA

Revista Interdisciplinaria de Humanidades, Educación, Ciencia y Tecnología

Año V. Vol. V. №1. Edición Especial. 2019

Hecho el depósito de ley: pp201602FA4721

ISSN-L: 2542-3029; ISSN: 2610-802X

Universidad Nacional Experimental Francisco de Miranda (UNEFM). Santa Ana de Coro. Venezuela

Lucía Noemí Morocho Malla; Cecilia Ivonne Narváez Zurita; Juan Carlos Erazo Alvarez

\begin{tabular}{|c|c|c|c|c|c|c|c|c|c|c|c|}
\hline $\begin{array}{l}\text { operario de apilador } \\
\text { eléctrico }\end{array}$ & & & & & & 290,00 & & & 290,00 & 310,00 & $6.45 \%$ \\
\hline \multirow{2}{*}{$\begin{array}{l}\text { Costos Indirectos } \\
\text { Mano de Obra } \\
\text { Indirecta }\end{array}$} & 90,00 & 0,00 & 0,00 & 0,00 & 549,50 & 290,00 & 130,00 & 140,00 & $1,199.50$ & $1,244.50$ & $3.62 \%$ \\
\hline & 90,00 & 0,00 & 0,00 & 0,00 & 100,00 & 60,00 & 110,00 & 110,00 & 470,00 & 495,00 & $5.05 \%$ \\
\hline Jefe Mecánico & & & & & 100,00 & 60,00 & & & 160,00 & 170,00 & $5.88 \%$ \\
\hline Auxiliar de servicios & 90,00 & & & & & & & & 90,00 & 95,00 & $5.26 \%$ \\
\hline CIF & 0,00 & 0,00 & 0,00 & 0,00 & 449,50 & 230,00 & 20,00 & 30,00 & 729,50 & 749,50 & $2.67 \%$ \\
\hline insumos varios & & & & & 100,00 & 80,00 & 20,00 & 30,00 & 230,00 & 240,00 & $4.17 \%$ \\
\hline \multirow[t]{2}{*}{ Costo Total } & 350,00 & $4.485,00$ & 64,00 & 992,00 & 869,50 & 580,00 & 130,00 & 140,00 & $7.610,50$ & $7.730,50$ & $1.55 \%$ \\
\hline & & & & & Unidad & 19.200 & \multicolumn{2}{|c|}{ Costo Unitario } & 0,396 & 0,403 & $2 \%$ \\
\hline
\end{tabular}

Al observar la tabla 9 y 10 se verifica la aplicación del sistema de gestión de costos $\mathrm{ABC} / \mathrm{ABM}$, el costo total y unitario obtenido de los dos servicios ofertados por la empresa Loadfrans es menor ante el método tradicional, siendo un $3 \%$ sobre la carga suelta y un $2 \%$ por la carga paletizada; cabe indicar, que es preciso distribuir de forma estratégica las actividades que conllevan más recursos y tener un mayor control y efectividad en la obtención de los costos que intervienen en los dos servicios analizados, donde el porcentaje de variación relativa se acentúa en la Mano de Obra Directa y los Costos Indirectos. Una vez aplicado el nuevo sistema se identificara de manera correcta los costos pertenecientes a cada proceso, con optimización de tiempo, reducción del mal uso y desperdicio de recursos.

Implementación y seguimiento: el método de costos utilizado cambia el resultado del costo total y unitario que mantiene la empresa objeto de estudio, por lo que queda a criterio y decisión de sus directivos la implementación del modelo propuesto, y además su seguimiento y evaluación, determinando responsables para cada actividad.

Al efectuar un seguimiento y evaluación se considera ejecutar un análisis de la rentabilidad del servicio de la carga suelta y paletizada, por lo que se procede presentar una comparación entre el costo tradicional utilizado por la empresa y el sistema propuesto de costeo $\mathrm{ABC} / \mathrm{ABM}$, cargando al gasto el CIF excedido entre estos dos métodos, lo cual se demuestra en la tabla 11: 


\section{Tabla 11}

Margen de rentabilidad de carga paletizada y carga suelta

\begin{tabular}{lrrrr} 
& \multicolumn{2}{c}{ Carga paletizada } & \multicolumn{2}{c}{ Carga suelta } \\
\hline & ABC/ABM & $\begin{array}{c}\text { Costeo } \\
\text { Tradicional }\end{array}$ & ABC/ABM & $\begin{array}{r}\text { Costeo } \\
\text { Tradicional }\end{array}$ \\
Costo Unitario & 0,396 & 0,403 & 0,108 & 0,111 \\
$\begin{array}{l}\text { Precio de Venta } \\
\text { Utilidad }\end{array}$ & 0,440 & 0,440 & 0,130 & 0,130 \\
$\begin{array}{l}\text { CIF individual cargado al } \\
\text { gasto }\end{array}$ & $\mathbf{0 , 0 4 4}$ & $\mathbf{0 , 0 3 7}$ & $\mathbf{0 , 0 2 3}$ & $\mathbf{0 , 0 1 9}$ \\
$\begin{array}{l}\text { Utilidad Neta } \\
\text { \% relativo de incremento del }\end{array}$ & 0,000 & 0,006 & 0,000 & 0,004 \\
$\begin{array}{l}\text { margen utilidad entre costeo } \\
\text { tradicional y ABC/ABM }\end{array}$ & $\mathbf{0 , 0 4 4}$ & 0,031 & $\mathbf{0 , 0 2 3}$ & 0,015 \\
\hline
\end{tabular}

Ante el cálculo expuesto, Al aplicar el modelo de gestión de costos ABC/ABM ante el uso del costeo tradicional, observamos un incremento en la utilidad neta del $40.17 \%$ en la carga paletizada y $51,69 \%$ en la carga suelta; por tal razón, consideramos favorable la aplicación de la gestión de costos $\mathrm{ABC} / \mathrm{ABM}$ para este tipo de empresas de servicio.

\section{CONCLUSIONES}

La aplicación del modelo de gestión de costos en actividades $A B C / A B M$ realizado a la empresa "LOAFRANS" S.A. de la ciudad de Machala, presenta las siguientes conclusiones:

- La aplicación de este modelo permite verificar que los costos por los servicios portuarios son entre el 2 y $3 \%$ menores a los costos establecidos por la contabilidad de la empresa; por consiguiente, incrementando la rentabilidad de la misma.

- Ejecutar y aplicar el modelo de gestión de costos ABC/ABM permitirá a la empresa optimizar los procesos de planificación de costos, lo que ayuda a identificar costos innecesarios, que perjudican a la utilidad; de esta forma, añadir valor a los procesos y actividades que servirán de ventaja competitiva para la empresa al administrar eficientemente los costos. 
- Se propone a la empresa identificar y clasificar las actividades que realiza, con la finalidad que cada actividad se ejecute de forma correcta, respetando los debidos procesos y estableciendo los centros de costos para determinar el costo operacional con el propósito de brindar un servicio de calidad hacia sus clientes, todo esto conllevaría a la obtención de herramientas útiles para la toma de decisiones de sus directivos.

- La implementación del modelo de gestión basado en actividades ABC/ABM permitirá a la empresa mejorar su rentabilidad; puesto que, identificará los puntos medulares que obstaculizan la efectividad y calidad de los servicios brindados por la unidad de análisis.

- Existe una cultura organizacional moderna que permite la innovación y aplicación de nuevos modelos de gestión de costos como el ABC/ABM en las empresas, con el objetivo de incrementar sus ganancias y optimizar sus costos.

\section{REFERENCIAS CONSULTADAS}

1. Brewer, P., Garrison, R., \& Noreen, E. (2007). Contabilidad administrativa. México D.F.: Mc Graw Hill.

2. Caldera, J., y Ripoll, V. (2003). Factores de organización y comportamiento en los sistemas $A B C / A B M$ : Estado de la cuestión. Compendium: revista de investigación científica, 43-63.

Retrieved

from https://dialnet.unirioja.es/servlet/articulo?codigo=2955142

3. Chacón, G., Bustos, C., y Rojas, E. S. (2006). Los Procesos de Producción y la Contabilidad de Costos. Actualidad Contable Faces, 9(12), 16-26. Retrieved from http://www.redalyc.org/articulo.oa?id=25701203

4. Coronado, A. (2017). Propuesta de implementación de sistema de costos abc para los procesos de captación de clientes y prestación de servicios y su efecto en la rentabilidad de la empresa inicia, fútbol y valores asociación civil, Lima 2017. Lima: Universidad San Ignacio de Loyola. Retrieved from http://repositorio.usil.edu.pe/bitstream/USIL/3488/1/2017_CoronadoVillalobos.pdf 
Revista Interdisciplinaria de Humanidades, Educación, Ciencia y Tecnología

Año V. Vol. V. №1. Edición Especial. 2019

Hecho el depósito de ley: pp201602FA4721

ISSN-L: 2542-3029; ISSN: 2610-802X

Universidad Nacional Experimental Francisco de Miranda (UNEFM). Santa Ana de Coro. Venezuela

Lucía Noemí Morocho Malla; Cecilia Ivonne Narváez Zurita; Juan Carlos Erazo Alvarez

5. Cuervo, J., Duque, M., y Osorio, J. (2013). Costeo basado en actividades ABC Gestión basada en actividades ABM (Segunda ed.). ECOE EDICIONES. Retrieved from https://www.ecoeediciones.com/wp-content/uploads/2015/09/Costeobasado-en-actividades-ABC.pdf

6. Cuevas, C., Chávez, G., Castillo, J., Marino, N., y Solarte, W. (2004). COSTEO ABC. ¿POR QUÉ Y CÓMO IMPLANTARLO? Universidad Icesi. Retrieved from http://www.scielo.org.co/scielo.php?pid=S0123$59232004000300003 \&$ script=sci_arttext\&tIng=pt

7. De la Hoz Granadillo, E. J., Fontalvo Herrera, T. J., \& Morelos Gómez, J. (2014). Evaluación del comportamiento de los indicadores de productividad y rentabilidad financiera del sector petróleo y gas en Colombia mediante el análisis discriminante. Contaduría y Administración, 59(4), 167-191. Retrieved from https://reader.elsevier.com/reader/sd/pii/S0186104214701597?token=C694BE89 E51809632C70279350238353D3DF5D9B61F046031084164980E3FD276CA1A 77B23BEA9DDD90EE1EBEC0C062B

8. Flores, M., Narváez, C., \& Erazo, J. (2109). Costos ABC/ABM como herramienta de gestión empresarial. Caso empresa PlanERP Cía. Ltda. Visionario Digital, 114135. doi:https://doi.org/10.33262/visionariodigital.v3i2.1.549

9. García, D., Marín, S., y Martínez, F. J. (2006). La contabilidad de costos y rentabilidad en la Pyme. Contaduría y Administración, 39-59. Retrieved from http://www.redalyc.org/articulo.oa?id=39521803

10. García, J. (2008). Contabilidad de Costos. México: McGraw-Hill. Retrieved from http://www.universidadcultural.com.mx/online/claroline/backends/download.php?u $\mathrm{rl}=$ LONPTIRBQKIMSURBRF9ERV9DT1NUT1NfREVfSIVBTI9HQVJDSUFfQ09MS U4ucGRm\&cidReset=true\&cidReq=LGS3

11. García, J. (2014). Cost accounting (Fourth Edition ed.). Mexico, DF: McGraw-Hill Interamericana Editores.

12. Hoz, B., Ferrer, M., y Hoz, A. (2008). Indicadores de rentabilidad: herramientas para la toma decisiones financieras en hoteles de categoría media ubicados en Maracaibo. Revista de Ciencias Sociales (Ve), XIV(1), 88-109. Retrieved from http://www.redalyc.org/articulo.oa?id=28011673008

13. Lizana, L. (2010). Costeo Basado en Actividades (ABC). PRICEWATERHOUSECOOPERS. 
14. López, M., Gómez, A., y Marín, S. (2011). Sistema de costos ABC en la mediana empresa industrial mexicana. Cuadernos de Contabilidad, 12(30), 23-43. Retrieved from http://www.scielo.org.co/scielo.php?pid=S0123$14722011000100002 \&$ script=sci_arttext\&tIng=en

15. Morillo, M. (2002). Diseño de Sistemas de Costeo: Fundamentos Teóricos. Actualidad Contable Faces, 5(5), 7-22. Retrieved from http://www.redalyc.org/articulo.oa?id=25700507

16. Ortega Polanco, V. (2017). Gestión de la imagen corporativa de organizaciones universitarias desde el enfoque del marketing emocional. CIENCIAMATRIA, 3(5), 150-171. https://doi.org/10.35381/cm.v3i5.19

17. Pacheco, J. (2012). Gestión financiera con Excel. Lima: Editorial Macro. Retrieved from

https://books.google.com.ec/books?id=MwowDgAAQBAJ\&dq=indices+de+rentabi lidad+ROE+ROI+EVA\&source=gbs_navlinks_s

18. Pérez, O., y Tapanes, Y. (2009). Origen del Sistema de Gestión y Costos Basado En Actividades (ABC/ABM). Avanzada Científica, 12(1), 8. Retrieved from https://dialnet.unirioja.es/servlet/articulo?codigo $=5074421$

19. Ripoll Feliu, V., Caldera, J., y Vega Aparicio, Y. (2006). Percepción de la Dirección sobre los factores que influyen en la puesta en práctica del sistema $A B C / A B M$ : Un Estudio Cualitativo. Compendium: revista de investigación científica, 33-53. Retrieved from https://www.redalyc.org/articulo.oa?id=88001603

20. Rodríguez, E. (2008). "Activity Based Costing abc" costos basados en actividades, su aplicación a la gestión de una institución financiera "banca universal". Caracas: Universidad Católica Andrés Bello. Retrieved from http://biblioteca2.ucab.edu.ve/anexos/biblioteca/marc/texto/AAR2324.pdf

21. Rodríguez, J. (2010). El actual estado del arte en los Sistemas ABC/M: Un análisis e interpretación de la literatura internacional. Revista Iberoamericana de Contabilidad, 1-20. Retrieved from http://www.observatorioiberoamericano.org/ricg/N\%C2\%BA 15/Jorge Emilio Rodr\%C3\%ADguezMaeso.pdf

22. Rodríguez Rodríguez, S., Crúz, Y., \& Puertas, A. (2017). Indicadores para la comercialización de productos derivados de lotes caprinos (capra hircus), hacia una estrategia endógena de marketing en Colina - Zamora, Falcón, Venezuela.. 
Revista Arbitrada Interdisciplinaria Koinonía, 2(3), 198--212. Recuperado de http://fundacionkoinonia.com.ve/ojs/index.php/revistakoinonia/article/view/63/50

23. Sánchez, B. (2013). Implicancias del Método de Costeo ABC. Quipukamayoc, 21(39), 65-73. Retrieved from https://revistasinvestigacion.unmsm.edu.pe/index.php/quipu/article/view/6273/547 9

24.Zapata, P. (2015). Contabilidad de Costos (herramienta para la toma de decisiones). Colombia: Alfaomega.

\section{REFERENCES CONSULTED}

1. Brewer, P., Garrison, R., \& Noreen, E. (2007). Administrative Accounting Mexico City: Mc Graw Hill.

2. Caldera, J., and Ripoll, V. (2003). Organizational and behavioral factors in ABC / ABM systems: State of the matter. Compendium: scientific research journal, 43-63. Retrieved from https://dialnet.unirioja.es/servlet/articulo?codigo=2955142

3. Chacón, G., Bustos, C., and Rojas, E. S. (2006). Production Processes and Cost Accounting. Accounting Faces, 9 (12), 16-26. Retrieved from http://www.redalyc.org/articulo.oa?id=25701203

4. Coronado, A. (2017). Proposal for the implementation of an abc cost system for the processes of customer acquisition and service provision and its effect on the profitability of the company starts, football and civil association values, Lima 2017. Lima: Universidad San Ignacio de Loyola. Retrieved from http://repositorio.usil.edu.pe/bitstream/USIL/3488/1/2017_Coronado-

Villalobos.pdf

5. Cuervo, J., Duque, M., and Osorio, J. (2013). Costs based on ABC activities Management based on ABM activities (Second ed.). ECOE EDITIONS. Retrieved from https://www.ecoeediciones.com/wp-content/uploads/2015/09/Costeobasado-en-actividades-ABC.pdf

6. Cuevas, C., Chavez, G., Castillo, J., Marino, N., and Solarte, W. (2004). ABC COST. WHY AND HOW TO IMPLEMENT IT? Icesi University. Retrieved from http://www.scielo.org.co/scielo.php?pid=S0123-

59232004000300003\&script=sci_arttext\&tlng=pt 


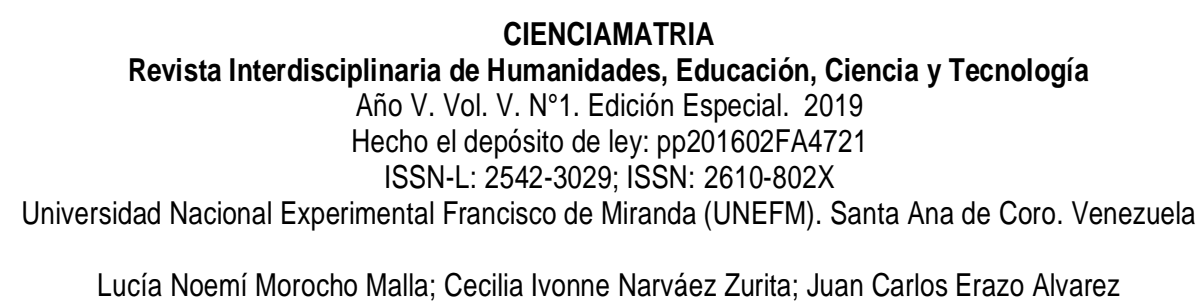

7. De la Hoz Granadillo, E. J., Fontalvo Herrera, T. J., \& Morelos Gómez, J. (2014). Evaluation of the performance of the indicators of productivity and financial profitability of the oil and gas sector in Colombia through discriminant analysis. Accounting and Administration, 59 (4), 167-191. Retrieved from https://reader.elsevier.com/reader/sd/pii/S0186104214701597?token=C694BE89 E51809632C70279350238353D3DF5D9B61F046031084164980E3FD276CA1A 77B23BEA9DD090C1BBB62B

8. Flores, M., Narváez, C., \& Erazo, J. (2109). ABC / ABM costs as a business management tool. Case of PlanERP Cía. Ltda. Digital Visionary, 114-135. doi: https: //doi.org/10.33262/visionariodigital.v3i2.1.549

9. García, D., Marín, S., and Martínez, F. J. (2006). Cost and profitability accounting in SMEs. Accounting and Administration, 39-59. Retrieved from http://www.redalyc.org/articulo.oa?id=39521803

10.García, J. (2008). Cost accounting. Mexico: McGraw-Hill. Retrieved from http://www.universidadcultural.com.mx/online/claroline/backends/download.php?u $\mathrm{rl}=$ LONPTIRBQkIMSURBRF9ERV9DT1NUT1NfREVfSIVBTI9HQVJDSUFfQ09MS U4ucGRm\&cidReset=true\&cSS =

11. García, J. (2014). Cost accounting (Fourth Edition ed.). Mexico City: McGraw-Hill Interamericana Editores.

12. Hoz, B., Ferrer, M., and Hoz, A. (2008). Profitability indicators: tools for making financial decisions in middle-class hotels located in Maracaibo. Journal of Social Sciences (Ve), XIV (1), 88-109. Retrieved from http://www.redalyc.org/articulo.oa?id=28011673008

13. Lizana, L. (2010). Activity Based Costing (ABC). PRICEWATERHOUSECOOPERS.

14. López, M., Gómez, A., and Marín, S. (2011). ABC cost system in the medium-sized Mexican industrial company. Accounting Notebooks, 12 (30), 23-43. Retrieved from http://www.scielo.org.co/scielo.php?pid=S0123$14722011000100002 \&$ script=sci_arttext\&tIng=en

15. Morillo, M. (2002). Design of Costing Systems: Theoretical Foundations. Accounting Faces, 5 (5), 7-22. Retrieved from http://www.redalyc.org/articulo.oa?id=25700507 


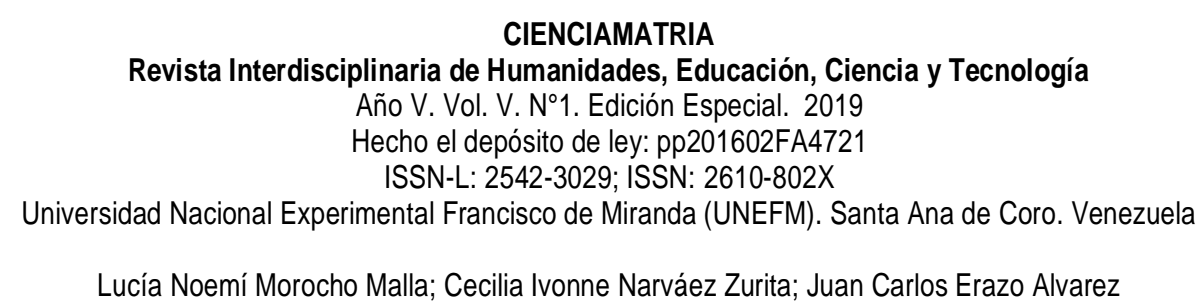

16. Ortega Polanco, V. (2017). Gestión de la imagen corporativa de organizaciones universitarias desde el enfoque del marketing emocional. CIENCIAMATRIA, 3(5), 150-171. https://doi.org/10.35381/cm.v3i5.19

17. Pacheco, J. (2012). Financial management with Excel. Lima: Editorial Macro. Retrieved from https://books.google.com.ec/books?id=MwowDgAAQBAJ\&dq=indices+de+rentabi lidad+ROE+ROI+EVA\&source=gbs_navlinks_s

18. Pérez, O., and Tapanes, Y. (2009). Origin of the Activity Based Management and Costs System (ABC / ABM). Advanced Scientific, 12 (1), 8. Retrieved from https://dialnet.unirioja.es/servlet/articulo?codigo $=5074421$

19. Ripoll Feliu, V., Caldera, J., and Vega Aparicio, Y. (2006). Management's perception of the factors that influence the implementation of the $A B C$ / $A B M$ system: A Qualitative Study. Compendium: scientific research journal, 33-53. Retrieved from https://www.redalyc.org/articulo.oa?id=88001603

20. Rodriguez, E. (2008). "Activity Based Costing abc" activity-based costs, its application to the management of a "universal banking" financial institution. Caracas: Andrés Bello Catholic University. Retrieved from http://biblioteca2.ucab.edu.ve/anexos/biblioteca/marc/texto/AAR2324.pdf

21. Rodríguez, J. (2010). The current state of the art in ABC / M Systems: An analysis and interpretation of international literature. Iberoamerican Journal of Accounting, 1-20. Retrieved from http://www.observatorioiberoamericano.org/ricg/N\%C2\%BA 15/Jorge Emilio Rodr\%C3\%ADguezMaeso.pdf

22. Rodríguez Rodríguez, S., Crúz, Y., \& Puertas, A. (2017). Indicadores para la comercialización de productos derivados de lotes caprinos (capra hircus), hacia una estrategia endógena de marketing en Colina - Zamora, Falcón, Venezuela.. Revista Arbitrada Interdisciplinaria Koinonía, 2(3), 198--212. Recuperado de http://fundacionkoinonia.com.ve/ojs/index.php/revistakoinonia/article/view/63/50

23. Sánchez, B. (2013). Implications of the ABC Costing Method. Quipukamayoc, 21 (39), 65-73. Retrieved from https://revistasinvestigacion.unmsm.edu.pe/index.php/quipu/article/view/6273/547 9

24.Zapata, P. (2015). Accounting 


\section{CIENCIAMATRIA}

Revista Interdisciplinaria de Humanidades, Educación, Ciencia y Tecnología

Año V. Vol. V. №1. Edición Especial. 2019

Hecho el depósito de ley: pp201602FA4721

ISSN-L: 2542-3029; ISSN: 2610-802X

Universidad Nacional Experimental Francisco de Miranda (UNEFM). Santa Ana de Coro. Venezuela

Lucía Noemí Morocho Malla; Cecilia Ivonne Narváez Zurita; Juan Carlos Erazo Alvarez

(C2019 por los autores. Este artículo es de acceso abierto y distribuido según los términos y condiciones de la licencia Creative Commons Atribución-NoComercial-Compartirlgual 4.0 Internacional (CC BY-NCSA 4.0) (https://creativecommons.org/licenses/by-nc-sa/4.0/). 